\title{
PATTERN MATCHING IN THE TEXTRACT INFORMATION EXTRACTION SYSTEM
}

\author{
Tsuyoshi Kitani ${ }^{\dagger}$ Yoshio Eriguchi ${ }^{\dagger \dagger}$ Masami IIara $^{\dagger \dagger}$ \\ Center for Machine Translation \\ Carnegie Mellon University \\ Pittsburgh, P^ 15213
}

\begin{abstract}
In information extraction systems, pallem matchers are widely used to identify information of interest in a sentence. In this paper, pattern matching in the TEXTRACT information extraction system is described. It comprises a concept search which identifies key words representing a concept, and a template pattern search which identifics palterns of words and phrases. TTXXTRACT using the matcher performed well in the TIPSTER/MUC-5 evaluation. The patlern matching architecture is also suitable for rapid system development across different domains of the same language.
\end{abstract}

\section{INTRODUCTION}

In information extraction systems, finitestate pattern matchers are becoming popular as a means of identifying individual pieces of information in a sentence. Pattern matching systems for English texts are reported to be suitable for achieving a high level of performance with less effort, compared to full parsing architectures [Hobbs et al. 92]. Among seventeen systems presented in the l'ifth Message Understanding Conference (MUC-5), three systems used a pattern matcher as the main component for identifying patterns to be extracted [MUC-5 93]. A pattern matching architecture is appropriate for information extraction from texts in narrow domains since identifying information does not necessarily require full understanding of the text. The pattern matcher can extract information of inter-

\footnotetext{
†Visiting researcher from N'T'T Data Communications Systems Corp., email: tkitaniourd.nttdata.jp

${ }^{\dagger \dagger} \mathrm{N}^{\prime} \mathrm{T} T$ Data Communications Systems Corp.
}

est by locating specific expressions defined as key words and phrasal patterns obtained by corpus analysis.

This paper describes a pattern matching method that first identifies concepts in a sentence and then links critical pieces of information that mas to a pattern. The first step in pattern matching is a concept search applicd in the TIXTRACI system of the TIPSTER Japanese microelectronics and corporate joint ventures domains [Jacobs 93a], [Jacobs 931)]. In this step, key words representing a concept are searched for within a sentence. The second step is a template pattern search applied in the TTXTRACT joint ventures system. A complex pattern to be searched for usually consists of a few words and phrases, instead of just one word, as in the concept search. The template pattern scarch recognizes relationships between matched objects in the defined pattern as well as recognizing the concept itself.

From the viewpoints of system performance and portability across domains, the TIPSTER/MUC-5 cvaluation results suggest that pattern matching described in this paper is an appropriate architecture for information extraction from Japanese texts.

\section{TTPSTER/MUC-5 OVERVIEW}

The goal of the TIPSTER/MUC-5 project sponsored by ARPA is to capture information of interest from English and Japanese newspaper articles about microelectronics and corporate joint ventures. ${ }^{1}$ A system must fill a generic template with information taken

\footnotetext{
${ }^{1}$ Several $\Lambda \mathrm{RP} A$-sponsored sites formed the TIPSTFR information extraction project. 'The TIPSTT'R sites and other non-sponsored organizations participated in MUC-5.
} 
from the text in a fully antomated fashion. 'The template is composed of several objects, each containing several slots. Slots may have pointers as values, where pointers link related objects. Fixtracted information is expected to be stored in an object-oriented database ['TIPSTER 92].

In the microelectronics domain, in formation about four specific processes in semiconductor manufacturing for microchip fabrication is captured. 'They are layering, lithography, etching, and packaging processes. Jayering, lithography, and etching are wafer fabrication processes; packaging is part of the last stage of manufacturing. Fintities such as manufacturer, distributor, and user, in addition to detailed manufacturing information such as materials used and the microchip specifications such as wafer size and devico speed are also extracted in each process.

The joint ventures domain focuses on extracting entities, i.e. organizations, forming or dissolving joint venture relationships. The information to be extracted includes entity information such as location, nationality, personnel, and facilities, and joint venture information such as relationships, business activities, capital, and estimated revenue of the joint venture.

\section{TEXTRACT ARCHITECTURE}

'TIX'RAC'T' is an information extraction system developed as an optional system of the GF-CMU SIIOGUN system [Jacolbs 93a], [Jacobs 93b]. It processes the TIPSTLER Japanese domains of microelectronics and corporate joint ventures. The T'TXTR $\Lambda$ C $T$ microclectronics system comprises three majol' components: preprocessing, concept search, and template gencration. In addition to con. cept search, the 'TEXTRACT' joint ventures system performs a template patteru search. It is also equipped with a discourse processor, as shown in Fig. 1.

In the preprocessor, Japanese text is segmented into primitive words tagged with their parts of spech by a Japanese segmentor called MAJESTYY [Kitani and Mitamura 93], [Kitani 91]. 'Then, proper nouns, along with monetary, numeric, and temporal expressions

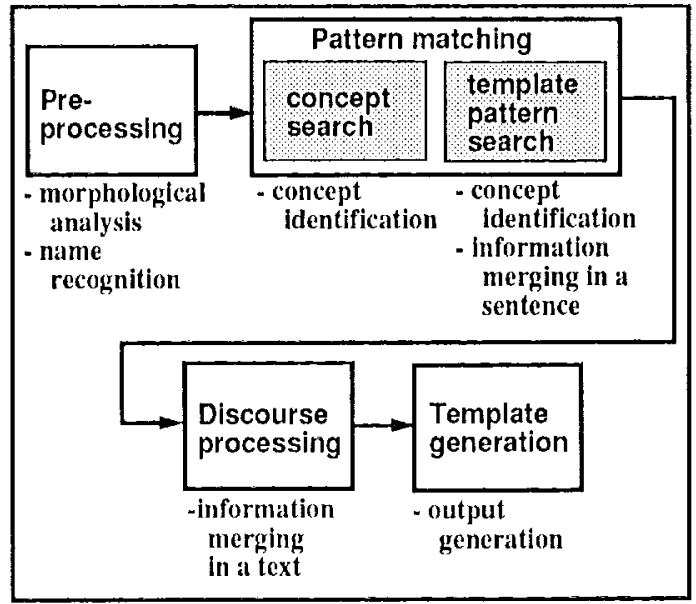

lig. 1: Architecture of the 'TFX'TRACI' joint ventures system

are identified by the name recognition module. 'lhe segments are grouped into units which are meaninghul in the pattern matching process [Kitani and Mitamuta 94]. Most strings to bo extracted directly from the text are identified lay MAIFSTY and the name recognizer in the preprocessor.

'The concept search and template pattern search modules both identily concepts in a sentence. The template pattern search also recognizes relationships within the identified information in the matched pattern. Details of the pattern matching process are described in the next section.

The discourse processor links information identilied at different stages of processing. First, implicit subjects, often used in Japanese sentences, are inherited from previous sentences, and second, company names aro given unicue numbers necessary to accurately recognize company relationships throughout the text [Kitani 94]. Concests identified during the pattern matching process are used to select an appropriate string and filler to go into a slot. Finally, the template generation process assembles the extracted information necessary to create the output described in section 2. 


\section{PATTERN MATCHING IN TEXTRACT}

\subsection{Concept search}

Key words representing the same concept are grouped into a list and used to recogni the concept in a sentence. The list is written in a simple format: (concept-name word1 word2 ...). For example, key words for recognizing a dissolved joint venture concept can be written in the following way:

\section{(DISSOLVED 提撨解消整理 消隇) \\ or \\ (DISSOLVED dissolve terminate cancel).}

The concept search module recognizes the concept when a word in the list exists in the sentence. Using such a simple word list sometimes generates an incorrect concept. For example, a dissolved concept is erronconsly identified from an expression "cancel a hotel reservation". Ilowever, when processing text in a narrow domain, concepts are often identified correctly from the simple list, since key words are usually used in a particular meaning of interest in the domain.

During the Japanese segmentation process in the preprocessor, a key word in the text tends to be divided into a few separate words by MAJESTY, when the word is not stored in the dictionary, For example, the compound

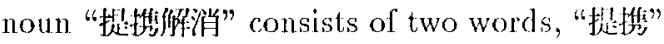
(joint venture) and "解消" (dissolve). It is segmented into the two individual nouns using the current MAJESTY dictionary. Thus, when the compound word "提按解消" is searched for in the segmented sentence, the concept search fails to identify it. To avoid this segmentation problem, adjacent nouns are automatically put together during the concept scarch process.

This process allows, by default, partial word matching between a key word and a word in

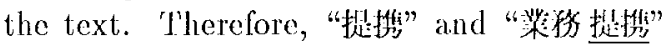
both meaning "a joint venture" can be identified by a single key word "扰携”. However, due to the nature of partial matching, the key word “シリコン” (Silicon) matches “-.-酸 化シリコン" (Silicon dioxide), which is a different type of film reported in the microolectronics domajn. This undesirable behavior can be avoided by attaching ">" to the beginning or " $<$ " to the end of key words. Thus,

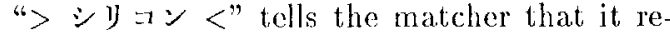
quires an exact word matching against a word in the text.

\subsection{Template pattern search}

\subsubsection{Template pattern matcher}

'The template pattern matcher identifies typical expressions to be extracted from the text that frequently appear in the corpus. 'The patterns are defined as pattern matching rules using regular expression.s.

The pattern matcher is a finite-state automaton similar to the pattern recognizer used in the MUC-4 FASTUS system developed at SRI [Hobbs et al. 92]. In TEX'IR $\Lambda \mathrm{CT}$, state transitions are driven by segmented words or grouped units from the preprocessor. The matcher identifies all possible patterns of interest in the text that match defined patterns. It must ignore unnecessary words in the pattern to perform successful pattern matching for various expressions.

\subsubsection{Pattern matching rules}

Fig. 2 shows a defined pattern in which an arbitrary string is represented as "@string" along with its corresponding linglish pattern. ${ }^{2}$ Specifically, a variable starting with "@CN $A M E$ " is called the company-name variable, used where a company name is expected to appear. For example, "@CNAML_PAR'INER_SU B.J" matches any string that likely includes at least one company name acting as a joint venture partner and functioning as a subject in the sentence.

The pattern “は |が:strict:P" tells the pattern matcher to identify the word, where "は" or "が" are grammatical particles that serve as subject case markers. The default type "strict" requires an exact string matcl, whereas "loose" allows a partial string matcli. Partial string matching is useful when compound words must be matched to a defined pattern. $\Lambda$ joint venture, “挻接: loose:VN", whose part of spech is verbal nominal, matiches compound words such as "芷桨 提携" (corporate joint venture) as well as "提 换" (joint venture).

\footnotetext{
2This Finglish pattern is used to capture expressions such as "XYZ Corp, created a joint venture with PQR
} 
(JointVenture 16

QCNAME_PARTNER_SUBJ

は |が:strict:P

QCNAME_PARTNER_WITH

$\varepsilon:$ strict:P

OSKIP

扰货: Loose:VN)

(a) A matching pattern for Japanese

(JointVenture1 3

OCNAME_PARTNER_SUBJ

create : $: V$

a joint venture: NP

with: :P

QCNAME_PARTNER_WITH)

(b) A matching pattern for English

Fig. 2: A matching pattern for (a) Japanese and (b) Jinglish

The first field in a pattern is the pattern name followed by the pattern number. 'The pattern number is nsed to decide whether or not a search within a given string is nocessary. 'lo assure efliciency with the pattern matcher, the field designated by the number should include the least frequent word in the entire pattern ("fut: for" for Japanese and "a joint venture" for Fnglish in this case).

\subsubsection{Pattern selection}

Approximately 150 patterns were used to extract various concepts in the Japanese joint ventures domain. Several patterns usually malch a single sentence. Moreover, siuce patterns are often searched using case markers such as "ば", “が", and "と”, which frecuently appear in Japanese texts, cven a single pattern can match the sentence in more than one way when several of the sane case markers exist in a sentence. However, since the template generator accepts only the best matched pattern, choosing a correctly matched pattern is important. The selection is done by applying three heuristic rules in the following order:

- select patterns that include the most number of matched company-name vari-

Inc:" ables in which there is at least one company name,

- select patterns that consume the fewest input segments (the shortest, string match), and

- select patterns that include the most number of variables and defined words.

Nuother important feature of the pattern matcher is that rules can be gromped according to their concept. $\Lambda$ rule name "Joint Venturel" in lig. 2, for example, represents a concept "JointVenture". Using this grouping, the best matched pattern can be selected from matched patterns of a particular concept group instead of choosing from all the matched patterns. This feature enables the discourse and template generation processes to look at the best information necessary whon filling in a. particular slot.

\section{EXAMPIE OF THE INFORMA- TION EXTRACTION PROCESS}

Ihis section describes how concepts and patterns identified by the matcher are used for template filling. Concepts are often useful to fill in the "set fill" (choice from a given set) slots. An entity type slot, for example, has four given choices: COMPANY, PliRSON, GOVFRNMTNT', and O'THFR. The matcher assigns concepts related to each entity type except O'THER. 'l'hus, from the given set, the output generator chooses an entity type corresponding to the identified concept. There are cases when discourse processing is necessary to link identified concepts and patterns. 'I'he following text: " $X$ Inc. created a joint venture with $Y$ Corp. last year. $X$ announced yesterday that it terminated the venture." is used to describe the extraction process illustrated in Fig. 3.

In the preprocessing, two company names in the first sentence "X Inc." and "Y Corp." are identilied either by MAJS'I'Y or the name recognizer. In the first sentence, the template pattern search locates the Joint Venturel pattern shown in Fig. 2. Now, the JOIN'L'Vlin'luRl' concept between "X Inc." and "Y Corp." is recognized. In the second sentence, 


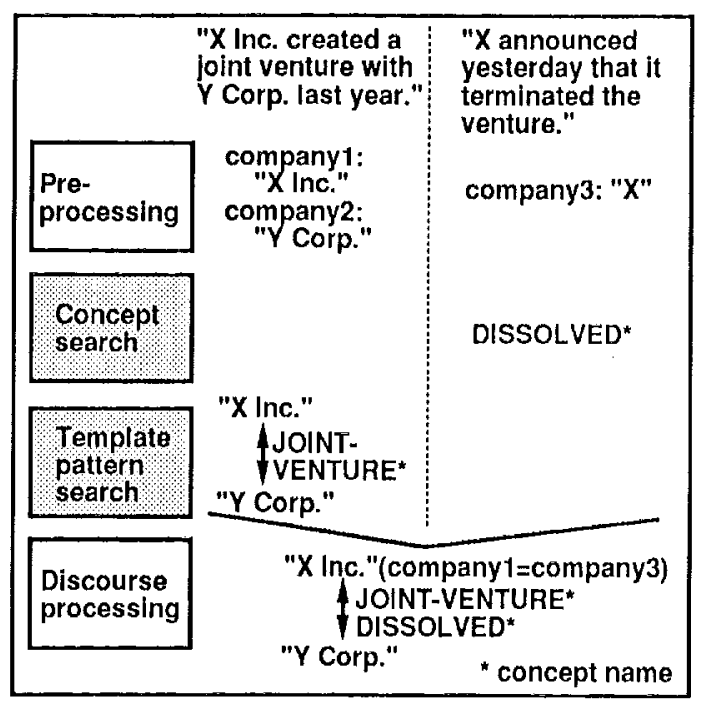

Fig. 3: Example of the information extraction process

the company name " $\mathrm{X}$ " is also identified by the preprocessor. ${ }^{3}$ Next, the concept "DISSOLVED" is recognized by the key word terminate in the concept search. (The key word list is shown in Section 4.1.) After sentencelevel processing, discourse processing recognizes that " $X$ " in the second sentence is a reference to "X Inc." found in the first sentence. Thus, the "DISSOLVLID" concept is joined to the joint venture relationship between "X Ine." and "Y Corp.". In this way, TEX'RACT recognizes that the two companies dissolved the joint venture.

Suppose that the second sentence is replaced with another sentence: "Shortly after, $X$ terminated a contract to supply rice to $Z$ Corp.". Although it does not mention the dissolved relationship nor anything about "Y Corp.", the system incorrectly recognizes the dissolved joint venture relationslip between "X Inc." and "Y Corp." due to the existence of the word terminate. When this undesirable matching is often seen, more complicated template patterns must be nsed instead of the simple word list. $A$ dissolved concept, for example, could be identified using the following template pattern:

\footnotetext{
"When it is an unknown word to the preprocesson' the discourse processor identifies it later.
}

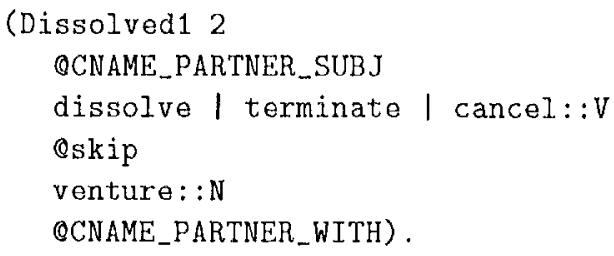

Then, discourse processing must check if companies identified in this pattern are the same as the current joint venture companies in order to recognize their dissolved relationship.

\section{OVERALL SYSTEM PERFOR- MANCE}

A total of 250 nowspaper articles, 100 about Japanese microelectronics and 150 about Japancse corporate joint ventures were provided by $A R P A$ for use in the TIPSTIR/MUC-5 system evaluation. Five microclectronics and six joint ventures systems were presented in the Japanese system evaluation at MUC-5. ${ }^{4}$ Scoring was done in a semi-antomatic mannor. The scoring program automatically compared the system output with answer templates created by human analysts, then, when a human decision was necessary, analysts instructed the scoring program whether the two strings in comparison were completely matched, partially matched, or ummatched. Finally, it calculated a.l overall score combined from all the newspaper article scores. Although various evaluation metrics were measured in the evaluation [Chinchor and Sundheim 93], only the following error and recall-precision metrics ase discussed in this paper. The basic scoring categories used are: correct (COR), partially correct (I'AR), incorrect (INC), missing (MIS), and spurious (SPU), counted as the number of pieces of information in the system output compared to the possible (answer) information.

(1) Lirror metrics

- Error per response fill (GRR):

$$
\frac{\text { wrong }}{\text { tolal }}=\frac{I N C+P A R / 2+M I S+S P U}{C O R+P A R+I N C+M I S+S P U}
$$

\footnotetext{
"I'hese numbers include 'I'PX'I'RAC'l, an optional system of CH-CMU, SHOCUN.
} 


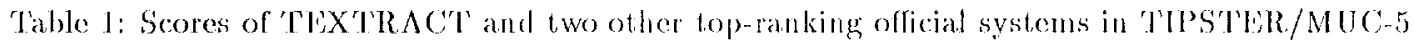

\begin{tabular}{|c|c|c|c|c|c|c|c|}
\hline domain & PRR & (UNI) & OVG & $\overline{S U B}$ & REC & PRLE & P\&R \\
\hline THX'TR $\Lambda$ C'I (JML) & 59 & 43 & 28 & 12 & 51 & $(i 3)$ & 56.3 \\
\hline System $\wedge(\mathrm{JMF})$ & 58 & 30 & 38 & 14 & 60 & 53 & 56.3 \\
\hline System B (JMF) & $6 \overline{5}$ & 54 & $\overline{24}$ & 12 & 40 & 66 & 50.4 \\
\hline TYXT'R $\triangle \mathrm{CT}(\mathrm{JV})$ & 50 & 31 & 23 & 12 & 60 & 68 & 63.8 \\
\hline System $\Lambda$ (JJV) & $5 \%$ & 36 & 27 & 12 & 57 & 64 & 60.1 \\
\hline System I3 (JJV) & 63 & 51 & 23 & 12 & 12 & 67 & 52.1 \\
\hline
\end{tabular}

'IEXT'RACT's scores submitted to MUC:-5 were anollicial.

JMFi, lapanese microclectronics domain

JJV: Japanese corporate joint ventures domain

- Undergeneration (UNI)):

$$
\frac{M I S}{\text { possible }}=\frac{M I S}{C O R+P A R+I N C+M I S}
$$

- Overgeneration (OV( $)$ :

$$
\frac{S P U}{\text { acutual }}=\frac{S P U}{C O R+P A R+T N G+S P U}
$$

- Substitution (SU1B):

$$
\frac{I N C+P A R / 2}{C O R+P A R+I N C}
$$

(2) Recall-precision metrics

- Recall (RLC):

$$
\frac{C O R+P A R / 2}{\text { possible }}
$$

- Precision (PRP):

$$
\frac{C O R+P A R / 2}{\text { aculual }}
$$

- P\&R F-measure (PRR):

$$
\frac{2 * R E C * P R E}{R L C+P R E}
$$

The error per response fill (likR) was the official measure of MUC -5 system performance. Secondary evaluation metrics were undergeneration (UND), overgeneration (OVG), and substitution (SUIB). 'The recall, precision, and F-measure metries were used as unoflicial met. rics for MUC;-5.

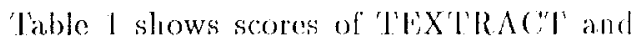
two other top-ranking official systems" taken

\footnotetext{
"LEXTRACT processed only Japhanes text whercas the two other systems processed both binglish and Japanese text.
}

from the TrPSTER/MUC,-5 system evaluation results[MUC:-593]. "THXTRACT performed equally with the lop-ranking systems in the two bapanese domains.

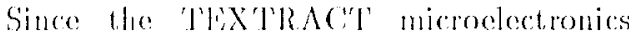
systen did not. include a template paltern search or discourse processor to help diflerenti ate between multiple semiconductor processes of the same kind, it reported only one object for cach kind of manufacturing process, even when multiple ob jects of the same kind existed in the article. This resulted in the lower scores in the microdectronics domain than those of the joint ventures domain.

This patheru matching architecture is highly portable across different domains of the same language. 'The TWXTRACT micmolectronics systen was developed in only three weeks by one person by simply replacing joint venture concepts and koy words with representative microelectronies concepts and liey words.

\section{CONCIUSTION}

In the Japanese microclectronics and corporate joint ventures domains, ITXTRACI performed erpally with the top-ranking ollicial systems at the 'TlPSTlR/MUG-5 system evaluation. Although performanee of paltern matching must be evaluated, the high performance of 'TWX'TRAO' suggests that the patcern matcher worked well in extracting information from the text. 'The pattern matcher

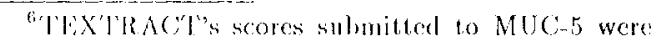
unofficial. It was seored officially after the conference. The official scores showed slight differences from nonfficial ones. 
has not been tested to languages other than Japanese. It is expected to work to other languages with some minor modifications given that the input is segmented into primitive words tagged with their parts of speech.

The TEXTRACT Japanese microelectronics system was developed in only three weeks by one person. In spite of its simplicity, it showed the high performance. This result also suggests that the pattern matching architecture is highly portable across similar domains of the same language, thus facilitating rapid system development. Developing and maintaining TEXTRACT's pattern matching based architecture is easier and less complex than that of a full parsing system, as experienced in the early stage of SHOGUN system development [Jacobs 93b].

Corpus analysis took about half of the development time, since only a KWIC (Key Word In Context) list and a word frequency tool were used to acquire the concept-word lists and the template patterns. Using good statistical corpus analysis tools will shorten the development time and promise a high performance. The tools should not only collect patterns of interest with context, but also give statistical data to show how well defined pat.terns are working when they are applied in the system.

At MUC-5 meeting, P\&R F-measure of one of the top-ranking systems was claimed to be close to the human performance [Jacobs 93b]. ${ }^{7}$ To match the system performance of a pattern matching system to human performance, the preprocessor must recognize expressions to be extracted at nearly $100 \%$ accuracy given that other components simply merge information and generate output.

\section{Acknowledgements}

The authors wish to express their appreciation to Jaime Carbonell, who provided the opportunity to pursue this research at the Center for Machine Translation, Carnegie Mellon University. Thanks are also due to Teruko Mitamura and Michael Mauldin for their many helpful suggestions.

\footnotetext{
The human performance was estimated to be recall and precision of about seventy to eighty.
}

\section{References}

[Chinchor and Sundheim 93] Chinchor, N. and Sundheim, B. (1993). MUC-5 Evaluation Metrics, Notebook of the Fifth Message Understanding Conference (MUC-5).

[Hobbs et al. 92] Hobbs, J., $\Lambda$ ppelt, D., et al. (1992). FASTUS: ^ System for Extracting Information from Natural-Language Text. SRI International, Technical Note No. 519.

[Jacobs 93a] Jacobs, P. (1993). TIPSTER/SIIOGUN 18-Month Progress Report. Notebook of the TIPSTER 18- Month Meeting.

[Jacobs 93b] Jacolos, P. (1993). GE-CMU: Description of the Shogun System Used for MUC-5. Notebook of the Fifth Message Understanding Conference (MUC-5).

[Kitani 91] Kitani, 'T. (1991). An OCR Post-processing Method for Jlandwritten Japanese Documents. In proceclings of Natural Language Processing Pacific Rim Symposium, pp. 38-45.

[Kitani and Mitamura 93] Kitani, 'I' and Mitamura, 'T. (1993). A Japanese Preprocessor for Syntactic and Semantic Parsing. In proceedings of Ninth IEET Conference on Artificial Intelligence for Applications, pp. 86-92.

[Kitani and Mitamura 94] Kitani, 'T. and Mitamura, 'T. (1994). An Accurate Morphological Analysis and Proper Name Identification for Japanese 'Text Processing. Journal of Information Processing Society of Japan, Vol. 35, No. 3, pp. 404 - 413.

[Kitani 94] Kitani, T'. (1994). Merging Information by Discourse Processing for Information Extraction. In procedings of Tenth IEEE Conference on Artificial Intelligence for Applications, pp. 412-418.

[MUC-5 93] (1993). System Descriptions. Notebook of the Fifh Message Understanding Conference (MUC-5).

[TIPSTRR 92] (1992). Joint Venture Template Fill Rules. Plenary Session Notebook of the TIPSTER 12-Month Meeting. 